\title{
STABILITY AND COMPACTNESS FOR COMPLETE $f$-MINIMAL SURFACES
}

\author{
XU CHENG, TITO MEJIA, AND DETANG ZHOU
}

\begin{abstract}
Let $\left(M, \bar{g}, e^{-f} d \mu\right)$ be a complete metric measure space with BakryÉmery Ricci curvature bounded below by a positive constant. We prove that in $M$ there is no complete two-sided $L_{f}$-stable immersed $f$-minimal hypersurface with finite weighted volume. Further, if $M$ is a 3-manifold, we prove a smooth compactness theorem for the space of complete embedded $f$-minimal surfaces in $M$ with the uniform upper bounds of genus and weighted volume, which generalizes the compactness theorem for complete self-shrinkers in $\mathbb{R}^{3}$ by Colding-Minicozzi.
\end{abstract}

\section{INTRODUCTION}

Recall that a self-shrinker (for mean curvature flow in $\mathbb{R}^{n+1}$ ) is a hypersurface $\Sigma$ immersed in the Euclidean space $\left(\mathbb{R}^{n+1}, g_{\text {can }}\right)$ satisfying that

$$
H=\frac{1}{2}\langle x, \nu\rangle,
$$

where $x$ is the position vector in $\mathbb{R}^{n+1}, \nu$ is the unit normal at $x$, and $H$ is the mean curvature of $\Sigma$ at $x$. Self-shrinkers play an important role in the study of singularity of mean curvature flow and have been studied by many people in recent years. We refer to [4, 5] and the references therein. In particular, Colding-Minicozzi [4] proved the following compactness theorem for self-shrinkers in $\mathbb{R}^{3}$.

Theorem 1 (4]). Given an integer $g \geq 0$ and a constant $V>0$, the space $\mathcal{S}(g, V)$ of smooth complete embedded self-shrinkers $\Sigma \subset \mathbb{R}^{3}$ with

- genus at most g,

- $\partial \Sigma=\emptyset$

- $\operatorname{Area}\left(B_{R}\left(x_{0}\right) \cap \Sigma\right) \leq V R^{2}$ for all $x_{0} \in \mathbb{R}^{3}$ and $R>0$

is compact. Namely, any sequence of these has a subsequence that converges in the topology of $C^{m}$ convergence on compact subsets for any $m \geq 2$.

In this paper, we extend Theorem 1 to the space of complete embedded $f$ minimal surfaces in a 3-manifold. A hypersurface $\Sigma$ immersed in a Riemannian manifold $(M, \bar{g})$ is called an $f$-minimal hypersurface if its mean curvature $H$ satisfies that, for any $p \in \Sigma$,

$$
H=\langle\bar{\nabla} f, \nu\rangle,
$$

Received by the editors March 6, 2013.

2010 Mathematics Subject Classification. Primary 58J50; Secondary 58E30.

The first and third authors were partially supported by CNPq and Faperj of Brazil.

The second author was supported by CNPq of Brazil. 
where $f$ is a smooth function defined on $M$ and $\bar{\nabla} f$ denotes the gradient of $f$ on $M$. Here are some examples of $f$-minimal hypersurfaces:

- $f \equiv C$, an $f$-minimal hypersurface is just a minimal hypersurface.

- self-shrinker $\Sigma$ in $\mathbb{R}^{n+1}$. $f=\frac{|x|^{2}}{4}$.

- Let $(M, \bar{g}, f)$ be a shrinking gradient Ricci solitons; i.e. after a normalization, $(M, \bar{g}, f)$ satisfies the equation $\overline{\operatorname{Ric}}+\bar{\nabla}^{2} f=\frac{1}{2} \bar{g}$ or equivalently the Bakry-Émery Ricci curvature $\overline{\operatorname{Ric}}_{f}:=\overline{\operatorname{Ric}}+\bar{\nabla}^{2} f=\frac{1}{2}$. We may consider $f$-minimal hypersurfaces in $(M, \bar{g}, f)$. In particular, the previous example: a self-shrinker $\Sigma$ in $\mathbb{R}^{n+1}$ is $f$-minimal in Gauss shrinking soliton $\left(\mathbb{R}^{n+1}, g_{\text {can }}, \frac{|x|^{2}}{4}\right)$.

- $M=\mathbb{H}^{n+1}(-1)$, the hyperbolic space. Let $r$ denote the distance function from a fixed point $p \in M$ and $f(x)=\operatorname{nar}^{2}(x)$, where $a>0$ is a constant. Now $\overline{\operatorname{Ric}_{f}} \geq n(2 a-1)$. The geodesic sphere of radius $r$ centered at $p$ is an $f$-minimal hypersurface if the radius $r$ satisfies $2 a r=\operatorname{coth} r$.

An $f$-minimal hypersurface $\Sigma$ can be viewed in two ways. One is that $\Sigma$ is $f$-minimal if and only if $\Sigma$ is a critical point of the weighted volume functional $e^{-f} d \sigma$, where $d \sigma$ is the volume element of $\Sigma$. The other one is that $\Sigma$ is $f$-minimal if and only if $\Sigma$ is minimal in the new conformal metric $\tilde{g}=e^{-\frac{2 f}{n}} \bar{g}$ (see Section 2 and Appendix). $f$-minimal hypersurfaces have been studied before as even more general stationary hypersurfaces for parametric elliptic functionals; see for instance the work of White [14] and Colding-Minicozzi [7.

We prove the following compactness result:

Theorem 2. Let $\left(M^{3}, \bar{g}, e^{-f} d \mu\right)$ be a complete smooth metric measure space and $\overline{R i c}_{f} \geq k$, where $k$ is a positive constant. Given an integer $g \geq 0$ and a constant $V>0$, the space $S_{g, V}$ of smooth complete embedded $f$-minimal surfaces $\Sigma \subset M$ with

- genus at most $g$,

- $\partial \Sigma=\emptyset$,

- $\int_{\Sigma} e^{-f} d \sigma \leq V$

is compact in the $C^{m}$ topology, for any $m \geq 2$. Namely, any sequence of $S_{g, V}$ has a subsequence that converges in the $C^{m}$ topology on compact subsets to a surface in $S_{g, V}$, for any $m \geq 2$.

Since the existence of the uniform scale-invariant area bound is equivalent to the existence of the uniform bound of the weighted area for self-shrinkers (see Remark 11 in Section [5), Theorem 21 implies Theorem 1]. Also, in [2], we will apply Theorem 2 to obtain a compactness theorem for the space of closed embedded $f$-minimal surfaces with the upper bounds of genus and diameter.

To prove Theorem 2, we need to prove a nonexistence result on $L_{f}$-stable $f$ minimal hypersurfaces, which is of independent interest.

Theorem 3. Let $\left(M^{n+1}, \bar{g}, e^{-f} d \mu\right)$ be a complete smooth metric measure space with $\overline{R i c}_{f} \geq k$, where $k$ is positive constant. Then there is no complete two-sided $L_{f}$-stable $f$-minimal hypersurface $\Sigma$ immersed in $(M, \bar{g})$ without boundary and with finite weighted volume (i.e. $\left.\int_{\Sigma} e^{-f} d \sigma<\infty\right)$, where $d \sigma$ denotes the volume element on $\Sigma$ determined by the induced metric from $(M, \bar{g})$. 
Here we explain briefly the meaning of $L_{f}$ stability. For an $f$-minimal hypersurface $\Sigma$, the $L_{f}$ operator is

$$
L_{f}=\Delta_{f}+|A|^{2}+\overline{\operatorname{Ric}}_{f}(\nu, \nu)
$$

where $\Delta_{f}=\Delta-\langle\nabla f, \nabla \cdot\rangle$ is the weighted Laplacian on $\Sigma$. In particular, for selfshrinkers, it is the so-called $L$ operator:

$$
L=\Delta+|A|^{2}-\frac{1}{2}\langle x, \nabla \cdot\rangle+\frac{1}{2} .
$$

$L_{f}$-stability of $\Sigma$ means that its weighted volume $\int_{\Sigma} e^{-f} d \sigma$ is locally minimal; that is, the second variation of its weighted volume is nonnegative for any compactly supported normal variation. We leave more details about the definition of $L_{f^{-}}$ stability and some of its properties to Section 2 and the Appendix.

For self-shrinkers in $\mathbb{R}^{n+1}$, Colding-Minicozzi [6] proved that

Theorem 4 ([6]). There are no L-stable smooth complete self-shrinkers without boundary and with polynomial volume growth in $\mathbb{R}^{n+1}$.

Since the first and third authors [3] of the present paper proved that for selfshrinkers, properness, the polynomial volume growth, and finite weighted volume are equivalent, Theorem 3 implies Theorem 4.

In this paper, we also discuss the relationship among the properness, polynomial volume growth and finite weighted volume of $f$-minimal submanifolds (Propositions 3. 4 and 5). We obtain their equivalence when the ambient space $(M, \bar{g}, f)$ is a shrinking gradient Ricci solitons, i.e. $\overline{\operatorname{Ric}}+\bar{\nabla}^{2} f=\frac{1}{2} \bar{g}$, with the condition that $f$ is a convex function with $|\bar{\nabla} f|^{2} \leq f$ (Corollary 1).

The rest of this paper is organized as follows: In Section 2 some definitions, notation and facts are given as preliminaries. In Section 3 we prove Propositions 3. 4 and 5. In Section 4 we prove Theorem 3. In Section 5 we prove Theorem 2 In the Appendix we calculate the second variation of the volume functional of $f$-minimal submanifolds and discuss some properties of $L_{f}$-stability for $f$-minimal submanifolds.

\section{Preliminaries}

In general, a smooth metric measure space, denoted by $\left(M^{m}, \bar{g}, e^{-f} d \mu\right)$, is an $m$-dimensional Riemannian manifold $\left(M^{m}, \bar{g}\right)$ together with a weighted volume form $e^{-f} d \mu$ on $M$, where $f$ is a smooth function on $M$ and $d \mu$ is the volume element induced by the metric $\bar{g}$. In this paper, unless otherwise specified, we denote by a bar all quantities on $(M, \bar{g})$, for instance by $\bar{\nabla}$ and $\overline{\text { Ric }}$, the Levi-Civita connection and the Ricci curvature tensor of $(M, \bar{g})$ respectively. For $\left(M, \bar{g}, e^{-f} d \mu\right)$, an important and natural tensor is the $\infty$-Bakry-Émery Ricci curvature tensor $\overline{\operatorname{Ric}}_{f}$ (for simplicity, Bakry-Émery Ricci curvature), which is defined by

$$
\overline{\operatorname{Ric}}_{f}:=\overline{\operatorname{Ric}}+\bar{\nabla}^{2} f,
$$

where $\bar{\nabla}^{2} f$ is the Hessian of $f$ on $M$. If $f$ is constant, $\overline{\operatorname{Ric}}_{f}$ is the Ricci curvature $\overline{\mathrm{Ric}}$ on $M$ respectively.

A Riemannian manifold with Bakry-Émery Ricci curvature bounded below by a positive constant has some properties similar to a Riemannian manifold with Ricci curvature bounded below by a positive constant. For instance, see the work of 
Wei-Wylie 13], Munteanu-Wang [1, 12 and the references therein. In this paper, we will use the following proposition by Morgan [10] (see also its proof in [13]).

Proposition 1. If a complete smooth metric measure space $\left(M, \bar{g}, e^{-f} d u\right)$ has $\overline{R i c}_{f} \geq k$, where $k$ is a positive constant, then $M$ has finite weighted volume (i.e. $\left.\int_{M} e^{-f} d \mu<\infty\right)$ and finite fundamental group.

Now, let $i: \Sigma^{n} \rightarrow M^{m}, n<m$, be an $n$-dimensional smooth immersion. Then $i:\left(\Sigma^{n} ; i^{*} \bar{g}\right) \rightarrow\left(M^{m}, \bar{g}\right)$ is an isometric immersion with the induced metric $i^{*} \bar{g}$. For simplicity, we still denote $i^{*} \bar{g}$ by $\bar{g}$ whenever there is no confusion. We will denote for instance by $\nabla$, Ric, $\Delta$ and $d \sigma$, the Levi-Civita connection, the Ricci curvature tensor, the Laplacian, and the volume element of $(\Sigma, \bar{g})$ respectively.

The function $f$ induces a weighted measure $e^{-f} d \sigma$ on $\Sigma$. Thus we have an induced smooth metric measure space $\left(\Sigma^{n}, \bar{g}, e^{-f} d \sigma\right)$.

The associated weighted Laplacian $\Delta_{f}$ on $(\Sigma, \bar{g})$ is defined by

$$
\Delta_{f} u:=\Delta u-\langle\nabla f, \nabla u\rangle .
$$

The second order operator $\Delta_{f}$ is a self-adjoint operator on the space of square integrable functions on $\Sigma$ with respect to the measure $e^{-f} d \sigma$ (however the Laplacian operator in general does not have this property).

The second fundamental form $A$ of $(\Sigma, \bar{g})$ is defined by

$$
A(X, Y)=\left(\bar{\nabla}_{X} Y\right)^{\perp}, \quad X, Y \in T_{p} \Sigma, p \in \Sigma,
$$

where $\perp$ denotes the projection to the normal bundle of $\Sigma$. The mean curvature vector $\mathbf{H}$ of $\Sigma$ is defined by $\mathbf{H}=\operatorname{tr} A=\sum_{i=1}^{n}\left(\bar{\nabla}_{e_{i}} e_{i}\right)^{\perp}$.

Definition 1. The weighted mean curvature vector of $\Sigma$ with respect to the metric $\bar{g}$ is defined by

$$
\mathbf{H}_{f}=\mathbf{H}+(\bar{\nabla} f)^{\perp} .
$$

The immersed submanifold $(\Sigma, \bar{g})$ is called $f$-minimal if its weighted mean curvature vector $\mathbf{H}_{f}$ vanishes identically, or equivalently if its mean curvature vector satisfies

$$
\mathbf{H}=-(\bar{\nabla} f)^{\perp} .
$$

Definition 2. The weighted volume of $(\Sigma, \bar{g})$ is defined by

$$
V_{f}(\Sigma):=\int_{\Sigma} e^{-f} d \sigma
$$

It is well known that $\Sigma$ is $f$-minimal if and only if $\Sigma$ is a critical point of the weighted volume functional. Namely, it holds that

Proposition 2. If $T$ is a compactly supported variational vector field on $\Sigma$, then the first variation formula of the weighted volume of $(\Sigma, \bar{g})$ is given by

$$
\left.\frac{d}{d t} V_{f}\left(\Sigma_{t}\right)\right|_{t=0}=-\int_{\Sigma}\left\langle T^{\perp}, \mathbf{H}_{f}\right\rangle_{\bar{g}} e^{-f} d \sigma
$$

On the other hand, an $f$-minimal submanifold can be viewed as a minimal submanifold under a conformal metric. Precisely, define the new metric $\tilde{g}=e^{-\frac{2}{n} f} \bar{g}$ on $M$, which is conformal to $\bar{g}$. Then the immersion $i: \Sigma \rightarrow M$ induces a metric $i^{*} \tilde{g}$ 
on $\Sigma$ from $(M, \tilde{g})$. In the following, $i^{*} \tilde{g}$ is still denoted by $\tilde{g}$ for simplicity. The volume of $(\Sigma, \tilde{g})$ is

$$
\tilde{V}(\Sigma):=\int_{\Sigma} d \tilde{\sigma}=\int_{\Sigma} e^{-f} d \sigma=\mathrm{V}_{f}(\Sigma)
$$

Hence Proposition 2 and (5) imply that

$$
\int_{\Sigma}\left\langle T^{\perp}, \tilde{\mathbf{H}}\right\rangle_{\tilde{g}} d \tilde{\sigma}=\int_{\Sigma}\left\langle T^{\perp}, \mathbf{H}_{f}\right\rangle_{\bar{g}} e^{-f} d \sigma
$$

where $d \tilde{\sigma}=e^{-f} d \sigma$ and $\tilde{\mathbf{H}}$ denote the volume element and the mean curvature vector of $\Sigma$ with respect to the conformal metric $\tilde{g}$ respectively.

Identity (6) implies that $\tilde{\mathbf{H}}=e^{\frac{2 f}{n}} \mathbf{H}_{f}$. Therefore $(\Sigma, \bar{g})$ is $f$-minimal in $(M, \bar{g})$ if and only if $(\Sigma, \tilde{g})$ is minimal in $(M, \tilde{g})$.

Now suppose that $\Sigma^{n}$ is a hypersurface immersed in $M^{n+1}$. Let $p \in \Sigma$ and $\nu$ be a unit normal at $p$. The second fundamental form $A$ and the mean curvature $H$ of $(\Sigma, \bar{g})$ are as follows:

$$
\begin{gathered}
A: T_{p} \Sigma \rightarrow T_{p} \Sigma, A(X)=\bar{\nabla}_{X} \nu, X \in T_{p} \Sigma, \\
H=\operatorname{tr} A=-\sum_{i=1}^{n}\left\langle\bar{\nabla}_{e_{i}} e_{i}, \nu\right\rangle .
\end{gathered}
$$

Hence the mean curvature vector $\mathbf{H}$ of $(\Sigma, \bar{g})$ satisfies $\mathbf{H}=-H \nu$. Define the weighted mean curvature $H_{f}$ of $(\Sigma, \bar{g})$ by $\mathbf{H}_{f}:=-H_{f} \nu$. Then

$$
H_{f}=H-\langle\bar{\nabla} f, \nu\rangle \text {. }
$$

Definition 3. A hypersurface $\Sigma$ immersed in $\left(M^{n+1}, \bar{g}, e^{-f} d \mu\right)$ with the induced metric $\bar{g}$ is called an $f$-minimal hypersurface if it satisfies

$$
H=\langle\bar{\nabla} f, \nu\rangle .
$$

For a hypersurface $(\Sigma, \bar{g})$, the $L_{f}$ operator is defined by

$$
L_{f}:=\Delta_{f}+|A|^{2}+\overline{\operatorname{Ric}}_{f}(\nu, \nu),
$$

where $|A|^{2}$ denotes the square of the norm of the second fundamental form $A$ of $\Sigma$.

The $L_{f}$-stability of $\Sigma$ is defined as follows:

Definition 4. A two-sided $f$-minimal hypersurface $\Sigma$ is said to be $L_{f}$-stable if for any compactly supported smooth function $\varphi \in C_{o}^{\infty}(\Sigma)$, it holds that

$$
-\int_{\Sigma} \varphi L_{f} \varphi e^{-f} d \sigma=\int_{\Sigma}\left[|\nabla \varphi|^{2}-\left(|A|^{2}+\overline{\operatorname{Ric}}_{f}(\nu, \nu)\right) \varphi^{2}\right] e^{-f} d \sigma \geq 0 .
$$

It is known that an $f$-minimal hypersurface $(\Sigma, \bar{g})$ is $L_{f}$-stable if and only if $(\Sigma, \tilde{g})$ is stable as a minimal surface with respect to the conformal metric $\tilde{g}=e^{-f} \bar{g}$. See more details in the Appendix of this paper.

In this paper, for closed hypersurfaces, we choose $\nu$ to be the outer unit normal. 


\section{Properness, polynomial volume Growth AND Finite WEIGHTED VOLUME OF $f$-MINIMAL HYPERSURFACES}

In [3], the first and third authors of the present paper proved that the finite weighted volume of a self-shrinker $\Sigma^{n}$ immersed in $\mathbb{R}^{m}$ implies it is properly immersed. In [9], Ding-Xin proved that a properly immersed self-shrinker must have the Euclidean volume growth. Combining these two results, it was proved in [3] that for immersed self-shrinkers, properness, polynomial volume growth and finite weighted volume are equivalent.

In this section we study the relationship among the properness, polynomial volume growth and finite weighted volume of $f$-minimal submaifolds, some of which will be used later in this paper.

Let $\Sigma$ be an $n$-dimensional submanifold in a complete manifold $M^{m}, n<m$. $\Sigma$ is said to have polynomial volume growth if, for a $p \in M$ fixed, there exist constants $C$ and $d$ so that for all $r \geq 1$,

$$
\operatorname{Vol}\left(B_{r}^{M}(p) \cap \Sigma\right) \leq C r^{d}
$$

where $B_{r}^{M}(p)$ is the extrinsic ball of radius $r$ centered at $p$ and $\operatorname{Vol}\left(B_{r}^{M}(p)\right)$ denotes the volume of $B_{r}^{M}(p) \cap \Sigma$. When $d=n$ in (9), $\Sigma$ is said to be of Euclidean volume growth.

Before proving the following Proposition 3, we recall an estimate implied by the Hessian comparison theorem (cf., for instance, [6], Lemma 7.1).

Lemma 1. Let $(M, \bar{g})$ be a complete Riemannian manifold with bounded geometry, that is, $M$ has sectional curvature bounded by $k\left(\left|K_{M}\right| \leq k\right)$, and injectivity radius bounded below by $i_{0}>0$. Then the distance function $r(x)$ satisfies

$$
\left|\bar{\nabla}^{2} r(V, V)-\frac{1}{r}\right| V-\left.\langle V, \bar{\nabla} r\rangle \bar{\nabla} r\right|^{2} \mid \leq \sqrt{k}
$$

for $r<\min \left\{i_{0}, \frac{1}{\sqrt{k}}\right\}$ and any unit vector $V \in T_{x} M$.

Using this estimate we will prove

Proposition 3. Let $\Sigma^{n}$ be a complete noncompact $f$-minimal submanifold immersed in a complete Riemannian manifold $M^{m}$. If $\Sigma$ has finite weighted volume, then $\Sigma$ is properly immersed.

Proof. We argue by contradiction. Since the argument is local, we may assume that $(M, g)$ has bounded geometry. Suppose that $\Sigma$ is not properly immersed. Then there exist a number $2 R<\min \left\{i_{0}, \frac{1}{\sqrt{k}}\right\}$ and $o \in M$ so that $\bar{B}_{R}^{M}(o) \cap \Sigma$ is not compact in $\Sigma$, where $\bar{B}_{R}^{M}$ (o) denotes the closure of the (open) ball $B_{R}^{M}(o)$ in $M$ of radius $R$ centered at $o$. Then for any $a>0$, there is a sequence $\left\{p_{k}\right\}$ of points in $B_{R}^{M}(o) \cap \Sigma$ with $\operatorname{dist}_{\Sigma}\left(p_{k}, p_{j}\right) \geq a>0$ for any $k \neq j$. So $B_{\frac{a}{2}}^{\Sigma}\left(p_{k}\right) \cap B_{\frac{a}{2}}^{\Sigma}\left(p_{j}\right)=\emptyset$ for any $k \neq j$, where $B_{\frac{a}{2}}^{\Sigma}\left(p_{k}\right)$ and $B_{\frac{a}{2}}^{\Sigma}\left(p_{j}\right)$ denote the intrinsic balls in $\Sigma$ of the radius $\frac{a}{2}$, centered at $p_{k}$ and $p_{j}$ respectively. Choose $a<2 R$. Then $B_{\frac{a}{2}}^{\Sigma}\left(p_{j}\right) \subset B_{2 R}^{M}(o)$. If 
$p \in B_{\frac{a}{2}}^{\Sigma}\left(p_{j}\right)$, the extrinsic distance function $r_{j}(p)=\operatorname{dist}_{M}\left(p, p_{j}\right)$ from $p_{j}$ satisfies

$$
\begin{aligned}
\Delta r_{j} & =\sum_{i=1}^{n} \bar{\nabla}^{2} r_{j}\left(e_{i}, e_{i}\right)+\left\langle\mathbf{H}, \bar{\nabla} r_{j}\right\rangle \\
& \geq \frac{n}{r_{j}}-\frac{1}{r_{j}}\left|\nabla r_{j}\right|^{2}-n \sqrt{k}-\left\langle\bar{\nabla} f^{\perp}, \bar{\nabla} r_{j}\right\rangle \\
& \geq \frac{n}{r_{j}}-\frac{1}{r_{j}}\left|\nabla r_{j}\right|^{2}-c,
\end{aligned}
$$

where $c=n \sqrt{k}+\sup _{B_{2 R}^{M}(0)}|\bar{\nabla} f|$. Lemma 1 is used above. Hence

$$
\Delta r_{j}^{2} \geq 2 n-2 c r_{j} .
$$

Choosing $a \leq \min \left\{\frac{n}{2 c}, 2 R\right\}$, we have for $0<\mu \leq \frac{a}{2}$,

$$
\begin{aligned}
\int_{B_{\mu}^{\Sigma}\left(p_{j}\right)}\left(2 n-2 c r_{j}\right) d \sigma & \leq \int_{B_{\mu}^{\Sigma}\left(p_{j}\right)} \Delta r_{j}^{2} d \sigma \\
& =\int_{\partial B_{\mu}^{\Sigma}\left(p_{j}\right)}\left\langle\nabla r_{j}^{2}, \nu\right\rangle d \sigma \\
& \leq 2 \mu A(\mu),
\end{aligned}
$$

where $\nu$ denotes the outward unit normal vector of $\partial B_{\mu}^{\Sigma}\left(p_{j}\right)$ and $A(\mu)$ denotes the area of $\partial B_{\mu}^{\Sigma}\left(p_{j}\right)$. Using the co-area formula in (10), we have

$$
\int_{0}^{\mu}(n-c s) A(s) d s \leq \int_{0}^{\mu} \int_{d_{\Sigma}\left(p, p_{j}\right)=s}\left(n-c r_{j}\right) d \sigma \leq \mu A(\mu) .
$$

This implies

$$
(n-c \mu) V(\mu) \leq V^{\prime}(\mu),
$$

where $V(\mu)$ denotes the volume of $B_{\mu}^{\Sigma}\left(p_{j}\right)$. So

$$
\frac{V^{\prime}(\mu)}{V(\mu)} \geq \frac{n}{\mu}-c .
$$

Integrating (12) from $\varepsilon>0$ to $\mu$, we have

$$
\frac{V(\mu)}{V(\varepsilon)} \geq\left(\frac{\mu}{\varepsilon}\right)^{n} e^{-c(\mu-\varepsilon)} .
$$

Since $\lim _{s \rightarrow 0^{+}} \frac{V(s)}{s^{n}}=\omega_{n}$,

$$
V(\mu) \geq \omega_{n} \mu^{n} e^{-c \mu}
$$

Thus we conclude

$$
\int_{\Sigma} e^{-f} d \sigma \geq \sum_{j=1}^{\infty} \int_{B_{\frac{a}{2}}^{\Sigma}\left(p_{j}\right)} e^{-f} d \sigma \geq \inf _{B_{2 R}^{M}(o)}\left(e^{-f}\right) \sum_{j=1}^{\infty} \int_{B_{\frac{a}{2}}^{\Sigma}\left(p_{j}\right)} d \sigma=\infty .
$$

This contradicts the assumption of the finite weighted volume of $\Sigma$.

Proposition 4. Let $\left(M^{m}, \bar{g}, e^{-f} d \mu\right)$ be a complete smooth metric measure space with $\overline{R i c}_{f}=k$, where $k$ is a positive constant. Assume that $f$ is a convex function. If $\Sigma^{n}$ is a complete noncompact properly immersed $f$-minimal submanifold in $M$, then $\Sigma$ has finite weighted volume and Euclidean (hence polynomial) volume growth. 
Proof. Since $(M, \bar{g}, f)$ is a gradient shrinking Ricci soliton, it is well known that, by a scaling of the metric $\bar{g}$ and a translating of $f$, still denoted by $\bar{g}$ and $f$ respectively, we may normalize the metric so that $k=\frac{1}{2}$ and the following identities hold:

$$
\begin{aligned}
\bar{R}+|\bar{\nabla} f|^{2}-f & =0, \\
\bar{R}+\bar{\Delta} f & =\frac{m}{2}, \\
\bar{R} & \geq 0 .
\end{aligned}
$$

From these equations, we have that

$$
\bar{\Delta} f-|\bar{\nabla} f|^{2}+f=\frac{m}{2} \quad \text { and } \quad|\bar{\nabla} f|^{2} \leq f .
$$

It was proved by Cao and the third author [1 that there is a positive constant $c$ so that

$$
\frac{1}{4}(r(x)-c)^{2} \leq f(x) \leq \frac{1}{4}(r(x)+c)^{2}
$$

for any $x \in M$ with $r(x)=\operatorname{dist}_{M}(p, x) \geq r_{0}$, where $p$ is a fixed point in $M$ and $c, r_{0}$ are positive constants that depend only on $m$ and $f(p)$.

By (14), we know that $f$ is a proper function on $M$. Since $\Sigma$ is properly immersed in $M$ and $f$ is proper in $M,\left.f\right|_{\Sigma}$ is also a proper smooth function on $\Sigma$. Note that with the scaling metric and translating $f, \Sigma$ is still $f$-minimal. Hence

$$
\begin{aligned}
\Delta f-|\nabla f|^{2}+f & =\left(\bar{\Delta} f-\sum_{\alpha=n+1}^{m} f_{\alpha \alpha}-\left|\bar{\nabla} f^{\perp}\right|^{2}\right)-\left|\bar{\nabla} f^{\top}\right|^{2}+f \\
& =\bar{\Delta} f-|\bar{\nabla} f|^{2}+f-\sum_{\alpha=n+1}^{m} f_{\alpha \alpha} \\
& \leq \frac{m}{2}
\end{aligned}
$$

Also we have

$$
|\nabla f|^{2}=\left|\bar{\nabla} f^{\top}\right|^{2} \leq|\bar{\nabla} f|^{2} \leq f .
$$

By Theorem 1.1 of $[3, \Sigma$ has finite weighted volume and the Euclidean volume growth of the sub-level set of $f$ with respect to the scaling metric and the translating $f$, and hence with respect to the original metric and $f$. Moreover, by the estimate (14), we have that $\Sigma$ has the Euclidean volume growth.

Next we prove the following:

Proposition 5. Let $\left(M^{m}, \bar{g}, e^{-f} d \mu\right)$ be a complete smooth metric measure space with $\overline{R i c}_{f} \geq k$, where $k$ is a positive constant. Assume that $|\bar{\nabla} f|^{2} \leq 2 k f$. If $\Sigma^{n}$ is a complete submanifold (not necessarily $f$-minimal) with polynomial volume growth, then $\Sigma$ has finite weighted volume.

Proof. By a scaling of the metric, we may assume that $k=\frac{1}{2}$. The proof follows from an estimate of $f$. Munteanu-Wang [11] extended the estimate (14) to $\left(M^{m}, \bar{g}, e^{-f} d \mu\right)$ with $\overline{\operatorname{Ric}}_{f} \geq \frac{1}{2}$ and $|\bar{\nabla} f|^{2} \leq f$. Combining the assumption that $\Sigma$ 
has polynomial volume growth with the estimate (14), we have

$$
\begin{aligned}
\int_{\Sigma} e^{-f} d \sigma & =\int_{\Sigma \cap B_{r_{0}}^{M}(p)} e^{-f} d \sigma+\sum_{i=0}^{\infty} \int_{\Sigma \cap\left(B_{r_{0}+i+1}^{M}(p) \backslash B_{r_{0}+i}^{M}(p)\right)} e^{-f} d \sigma \\
& \leq C_{1} \operatorname{Vol}\left(\Sigma \cap B_{r_{0}}^{M}(p)\right)+C \sum_{i=0}^{\infty} e^{-\frac{1}{4}\left(r_{0}+i-c\right)^{2}} \operatorname{Vol}\left(\Sigma \cap B_{r_{0}+i+1}^{M}(p)\right) \\
& \leq C\left[r_{0}^{d}+\sum_{i=0}^{\infty} e^{-\frac{1}{4}\left(r_{0}+i-c\right)^{2}}\left(r_{0}+i+1\right)^{d}\right] \\
& <\infty
\end{aligned}
$$

By Propositions 3, 4 and 5, we have the following.

Corollary 1. Let $\left(M^{m}, \bar{g}, f\right)$ be a complete shrinking gradient Ricci soliton with $\overline{R i c}_{f}=\frac{1}{2}$. Assume that $f$ is a convex function. If $\Sigma$ is a complete $f$-minimal submanifold immersed in $M$, then for $\Sigma$ the properness, polynomial volume growth, and finite weighted volume are equivalent.

\section{Nonexistence of $L_{f}$ Stable $f$-Minimal hypersurfaces}

In this section, we prove Theorem 3 , which is a key to proving the compactness theorem in Section 5 .

Theorem 5 (Theorem 3). Let $\left(M, \bar{g}, e^{-f} d \mu\right)$ be a complete smooth metric measure space with $\overline{\operatorname{Ric}}_{f} \geq k$, where $k$ is a positive constant. Then there is no two-sided $L_{f}$-stable complete $f$-minimal hypersurface $\Sigma$ immersed in $(M, g)$ without boundary and with finite weighted volume (i.e. $\left.\int_{\Sigma} e^{-f} d \sigma<\infty\right)$.

Proof. We argue by contradiction. Suppose that $\Sigma$ is an $L_{f}$-stable complete $f$ minimal hypersurface immersed in $(M, g)$ without boundary and with finite weighted volume. Recall that a two-sided hypersurface $\Sigma$ is $L_{f}$-stable if the following inequality holds, that is, for any compactly supported smooth function $\varphi \in \mathcal{C}_{o}^{\infty}(\Sigma)$,

$$
\int_{\Sigma}\left[|\nabla \varphi|^{2}-\left(|A|^{2}+\overline{\operatorname{Ric}}_{f}(\nu, \nu)\right) \varphi^{2}\right] e^{-f} d \sigma \geq 0 .
$$

Observe that any closed hypersurface cannot be $L_{f}$-stable. This is because the assumption $\overline{\operatorname{Ric}}_{f} \geq k>0$ implies that (15) cannot hold for $\varphi \equiv c$ on $\Sigma$. Hence, $\Sigma$ must be noncompact.

Let $\eta$ be a nonnegative smooth function on $[0, \infty)$ satisfying

$$
\eta(s)=\left\{\begin{array}{llc}
1 & \text { if } & s \in[0,1) \\
0 & \text { if } \quad s \in[2, \infty)
\end{array}\right.
$$

and $\left|\eta^{\prime}\right| \leq 2$.

Fix a point $p \in \Sigma$ and let $r(x)=\operatorname{dist}_{\Sigma}(p, x)$ denote the (intrinsic) distance function on $\Sigma$. Define a sequence of functions $\varphi_{j}(x)=\eta\left(\frac{r(x)}{j}\right), j \geq 1$. Then 
$\left|\nabla \varphi_{j}\right|^{2} \leq 1$ for $j \geq 2$. Substituting $\varphi_{j}, j \geq 2$ for $\varphi$ in (15):

$$
\begin{aligned}
\int_{\Sigma}\left[\left|\nabla \varphi_{j}\right|^{2}\right. & \left.-\left(|A|^{2}+\overline{\operatorname{Ric}}_{f}(\nu, \nu)\right) \varphi_{j}^{2}\right] e^{-f} d \sigma \\
& \leq \int_{\Sigma}\left(\left|\nabla \varphi_{j}\right|^{2}-k \varphi_{j}^{2}\right) e^{-f} d \sigma \\
& =\int_{B_{2 j}^{\Sigma}(p) \backslash B_{j}^{\Sigma}(p)}\left|\nabla \varphi_{j}\right|^{2} e^{-f} d \sigma-\int_{B_{2 j}^{\Sigma}(p)} k \varphi_{j}^{2} e^{-f} d \sigma \\
& \leq \int_{B_{2 j}^{\Sigma}(p) \backslash B_{j}^{\Sigma}(p)} e^{-f} d \sigma-k \int_{B_{2 j}^{\Sigma}(p)} \varphi_{j}^{2} e^{-f} d \sigma \\
& \leq \int_{B_{2 j}^{\Sigma}(p) \backslash B_{j}^{\Sigma}(p)} e^{-f} d \sigma-k \int_{B_{2}^{\Sigma}(p)} e^{-f} d \sigma,
\end{aligned}
$$

where $B_{j}^{\Sigma}(p)$ is the intrinsic geodesic ball in $M$ of radius $j$ centered at $p$. Since $\Sigma$ has finite weighted volume, we have, when $j \rightarrow \infty$,

$$
\int_{B_{2 j}^{\Sigma}(p) \backslash B_{j}^{\Sigma}(p)} e^{-f} d \sigma \rightarrow 0 .
$$

Choosing $j$ large enough, we have that $\varphi_{j}$ satisfies

$$
\int_{\Sigma}\left(\left|\nabla \varphi_{j}\right|^{2}-\left(|A|^{2}+\overline{\operatorname{Ric}}_{f}(\nu, \nu)\right) \varphi_{j}^{2}\right) e^{-f} d \sigma<-\frac{k}{2} \int_{B_{2}^{\Sigma}(p)} e^{-f} d \sigma<0 .
$$

This contradicts the fact that $\Sigma$ is $L_{f}$-stable.

\section{Compactness of Complete $f$-minimal surfaces}

Before proving Theorem 2, we give some facts.

Wei-Wylie ([13, Theorem 7.3) used the mean curvature comparison theorem to give a distance estimate for two compact hypersurfaces $\Sigma_{1}$ and $\Sigma_{2}$ in a smooth metric measure space $\left(M, \bar{g}, e^{-f} d \mu\right)$ with $\overline{\operatorname{Ric}}_{f} \geq k$, where $k$ is a positive constant. Observe that for two complete properly immersed hypersurfaces $\Sigma_{1}$ and $\Sigma_{2}$, if at least one of them is compact, there is a minimizing geodesic segment joining $\Sigma_{1}$ and $\Sigma_{2}$ and realizing their distance. Hence the proof of Theorem $7.3[13$ can be applied to obtain the following.

Proposition 6. Let $\left(M, \bar{g}, e^{-f} d \mu\right)$ be an $(n+1)$-dimensional complete smooth metric measure space with $\overline{\text { Ric }}_{f} \geq k$, where $k$ is a positive constant. If $\Sigma_{1}$ and $\Sigma_{2}$ are two complete properly immersed hypersurfaces, at least one of which is compact, then the distance $d\left(\Sigma_{1}, \Sigma_{2}\right)$ satisfies

$$
d\left(\Sigma_{1}, \Sigma_{2}\right) \leq \frac{1}{k}\left(\max _{x \in \Sigma_{1}}\left|H_{f}^{\Sigma_{1}}(x)\right|+\max _{x \in \Sigma_{2}}\left|H_{f}^{\Sigma_{2}}(x)\right|\right),
$$

where $H_{f}^{\Sigma_{i}}, i=1,2$, denotes the weighted mean curvatures of $\Sigma_{i}$ respectively.

Corollary 2. Let $\left(M, \bar{g}, e^{-f} d \mu\right)$ be as in Proposition 6. Then there is a closed ball $\bar{B}^{M}$ of $M$ satisfying that any complete properly immersed $f$-minimal hypersurface $\Sigma$ must intersect it.

Proof. Fix $p \in M$ and a geodesic sphere $S_{r}^{M}(p)$ of $M$. By Proposition 6 .

$$
d\left(S_{r}^{M}(p), \Sigma\right) \leq \frac{1}{k} \max _{x}\left|H_{f}^{S_{r}^{M}(p)}(x)\right|=C,
$$


where $C$ is independent of $\Sigma$. Therefore there is a closed ball $\bar{B}^{M}$ of $M$ with radius big enough so that any $\Sigma$ must intersect it.

We need the following fact:

Proposition 7. Let $M$ be a simply connected Riemannian manifold. If a hypersurface $\Sigma$ is complete, not necessarily connected, properly embedded, and has no boundary, then every component of $\Sigma$ separates $M$ into two components and thus is two-sided. Therefore $\Sigma$ has a globally defined unit normal.

Proof. Suppose $\Sigma_{j}$ is a component of $\Sigma$. By contrast, $M \backslash \Sigma_{j}$ has one component. Since $\Sigma$ is a properly embedded $f$-minimal hypersurface, for any $p \in \Sigma_{j}$ there is a neighborhood $W$ of $p$ in $M$ so that $W \cap \Sigma_{j}=W \cap \Sigma$ only has one piece (i.e. it is a graph above a connected domain in the tangent plane of $p$ ). Thus we have a simply closed curve $\gamma$ passing $p$, transversal to $\Sigma_{j}$ at $p$, and $\Sigma_{j} \cap \gamma=p$. Since $M$ is simply connected, we have a disk $D$ with the boundary $\gamma$. Again since $\Sigma$ is proper, the intersection of $\Sigma_{j}$ with $\partial D=\gamma$ cannot be one point, which is a contradiction.

Combining Proposition 3 in Section 3 with Proposition [7, we obtain

Proposition 8. Let $\left(M, \bar{g}, e^{-f} d \mu\right)$ be a simply connected complete smooth metric measure space. If a complete $f$-minimal hypersurface has finite weighted volume, then every component of $\Sigma$ separates $M$ into two components and thus is two-sided. Therefore $\Sigma$ has a globally defined unit normal.

We will take the same approach as in Colding-Minicozzi's paper 4 to prove Theorem 2, a smooth compactness theorem for complete $f$-minimal surfaces. First we recall a well known local singular compactness theorem for embedded minimal surfaces in a Riemannian 3-manifold.

Proposition 9 (cf. [4, Proposition 2.1). Given a point $p$ in a Riemannian 3manifold $M$, there exists an $R>0$ such that the following holds: Let $\Sigma_{j}$ be embedded minimal surfaces in $B_{2 R}(p) \subset M$ with $\partial \Sigma_{j} \subset \partial B_{2 R}(p)$. If each $\Sigma_{j}$ has area at most $V$ and genus at most $g$ for some fixed $V, g$, then there exist a finite collection of points $x_{k}$, a smooth embedded minimal surface $\Sigma \subset B_{R}(p)$ with $\partial \Sigma \subset \partial B_{R}(p)$ and a subsequence of $\left\{\Sigma_{j}\right\}$ that converges in $B_{R}(p)$ (with finite multiplicity) to $\Sigma$ away from the set $\left\{x_{k}\right\}$.

Here and in the following, we denote by $B_{R}$ the ball $B_{R}^{M}$ in $M$ for simplicity.

It is known that $\Sigma$ is $f$-minimal with respect to metric $\bar{g}$ if and only if $\Sigma$ is minimal with the conformal metric $\tilde{g}=e^{-f} g$ (see Appendix). Using this fact and applying Proposition 9, we may prove a global singular compactness theorem for $f$-minimal surfaces.

Proposition 10. Let $M$ be a complete 3 -manifold and $\left(M, \bar{g}, e^{-f} d \mu\right)$ a smooth metric measure space. Suppose that $\Sigma_{i} \subset M$ is a sequence of smooth complete embedded $f$-minimal surfaces with genus at most $g$, without boundary, and with weighted area at most $V$, i.e.

$$
\int_{\Sigma_{i}} e^{-f} d \sigma \leq V<\infty
$$

Then there are a subsequence, still denoted by $\Sigma_{i}$, a smooth embedded complete nontrivial $f$-minimal surface $\Sigma \subset M$ without boundary, and a locally finite collection 
of points $\mathcal{S} \subset \Sigma$ so that $\Sigma_{i}$ converges smoothly (possibly with multiplicity) to $\Sigma$ off of $\mathcal{S}$. Moreover, $\Sigma$ satisfies $\int_{\Sigma} e^{-f} d \sigma \leq V$ and is properly embedded.

Here a set $\mathcal{S} \subset M$ is said to be locally finite if $B_{R}(p) \cap \mathcal{S}$ is finite for every $p \in M$ and for all $R>0$.

Proof. Consider the conformal metric $\tilde{g}=e^{-f} \bar{g}$ on $M$. For a point $p \in M$, let $\tilde{B}_{2 R}(p) \subset M$ denote the ball in $(M, \tilde{g})$ of radius $2 R$ centered at $p$. Then the area of $\tilde{B}_{2 R}(p) \cap \Sigma_{j}$ satisfies

$$
\widetilde{\operatorname{Area}}\left(\tilde{B}_{2 R}(p) \cap \Sigma_{j}\right) \leq \int_{\Sigma_{j}} d \tilde{\sigma}=\int_{\Sigma_{j}} e^{-f} d \sigma \leq V .
$$

Also, it is clear that the genus of $\tilde{B}_{2 R}(p) \cap \Sigma_{j}$ remains at most $g$. Then by Proposition 9. there exist an $R>0$ and a finite collection of points $x_{k}$, a smooth embedded minimal surface $\Sigma \subset \tilde{B}_{R}(p)$, with $\partial \Sigma \subset \partial \tilde{B}_{R}$ and a subsequence of $\left\{\Sigma_{j}\right\}$ that converges in $\tilde{B}_{R}(p)$ (with finite multiplicity) to $\Sigma$ away from the set $\left\{x_{k}\right\}$.

Let $\left\{\tilde{B}_{R_{i}}\left(p_{i}\right)\right\}$ be a countable cover of $(M, \tilde{g})$ of small balls such that $\left\{\tilde{B}_{2 R_{i}}\left(p_{i}\right)\right\}$ is still a cover of $(M, \tilde{g})$. On each $\tilde{B}_{2 R_{i}}\left(p_{i}\right)$, applying the previous local convergence and then passing to a diagonal subsequence, we obtain that there are a subsequence of $\Sigma_{i}$, still denoted by $\Sigma_{i}$, a smooth embedded minimal surface $\Sigma$ (with respect to the metric $\tilde{g}$ ) without boundary, and a locally finite collection of points $\mathcal{S} \subset \Sigma$ so that $\Sigma_{i}$ converges smoothly (possibly with multiplicity) to $\Sigma$ off of $\mathcal{S}$. Since $\Sigma$ has no boundary, it is complete in the original metric $\bar{g}$. Thus we obtain the smooth convergence of the subsequence to the smooth embedded complete $f$-minimal surface $\Sigma$ off of $\mathcal{S}$.

By Corollary 2, $\Sigma$ is nontrivial. The convergence of $\Sigma_{i}$ to $\Sigma$ and (17) imply $\int_{\Sigma} e^{-f} d \sigma \leq V$. By Proposition $[3$, $\Sigma$ is properly embedded.

We need to show that the convergence is smooth across the points in $\mathcal{S}$. To prove this, we need the following.

Proposition 11. Assume that the ambient manifold $M$ in Proposition 10 is simply connected. If the convergence of the sequence $\left\{\Sigma_{i}\right\}$ has multiplicity greater than one, then $\Sigma$ is $L_{f}$-stable.

Proof. By Proposition 8 , we know that $\Sigma_{i}$ and $\Sigma$ are orientable. We may have two ways to prove the proposition. The first is to use the known fact on minimal surfaces. It is known that (cf. [6], Appendix A) if the multiplicity of the convergence of a sequence of embedded orientable minimal surfaces in a simply connected 3manifold is not one, then the limit minimal surface is stable. Under the conformal metric $\tilde{g}$, a sequence $\left\{\Sigma_{i}\right\}$ of minimal surfaces converges to a smooth embedded orientable minimal surface $\Sigma$ and thus $\Sigma$ is stable. Also, the conclusion that $\Sigma$ is stable with respect to the conformal metric $\tilde{g}$ is equivalent to saying that $\Sigma$ is $L_{f}$-stable under the original metric $\bar{g}$ (see Appendix).

The second way is to prove it directly. We may prove that $L_{f}$ is the linearization of the $f$-minimal equation by a proof similar to the one in 4, Appendix A. By arguing as in Proposition 3.2 in [4, we can find a smooth positive function $u$ on $\Sigma$ satisfying

$$
L_{f} u=0 .
$$

This implies that $\Sigma$ is $L_{f}$-stable. 
Proof of Theorem 2. By the assumption on $\overline{\operatorname{Ric}}_{f}$ and Proposition 1, $M$ has finite fundamental group. After passing to the universal covering, we may assume that $M$ is simply connected. Given a sequence of smooth complete embedded $f$-minimal surfaces $\left\{\Sigma_{i}\right\}$ with genus $g, \partial \Sigma_{i}=\emptyset$, and the weighted area at most $V$, by Proposition 10 there is a subsequence, still denoted by $\left\{\Sigma_{i}\right\}$, that converges in the topology of smooth convergence on compact subsets to a smooth embedded complete $f$ minimal surface $\Sigma$ away from a locally finite set $\mathcal{S} \subset \Sigma$ (possibly with multiplicity). Moreover, the limit surface $\Sigma \subset M$ is complete, properly embedded, $\int_{\Sigma} e^{-f} d \sigma \leq V$, has no boundary and has a well-defined unit normal $\nu$. We also have the equivalent convergence under the conformal metric $\bar{g}$.

If $\mathcal{S}$ is not empty, Allard's regularity theorem implies that the convergence has multiplicity greater than one. Then by Proposition [11, we conclude that $\Sigma$ is $L_{f}$ stable. But Proposition 5 says that there is no such $\Sigma$. This contradiction implies that $\mathcal{S}$ must be empty. We complete the proof of the theorem.

Remark 1. For self-shrinkers, the condition that the scale-invariant uniform area bound exists (i.e. there is a uniform bound $V_{1}$ : $\operatorname{Area}\left(B_{R}\left(x_{0}\right) \cap \Sigma\right) \leq V_{1} R^{2}$ for all $x_{0} \in \mathbb{R}^{3}$ and $R>0$ ) implies that the uniform bound $V$ of weighted area (i.e. $\int_{\Sigma} e^{-f} d \sigma<V$ ) exists (cf. the proof of Proposition 5). The converse is also true by the conclusion that the entropy of a self-shrinker can be achieved by $F_{0,1}$ for selfshrinkers with polynomial volume growth (see Section 7 of [5]). Therefore Theorem 2 generalizes the result of Colding-Minicozzi (Theorem 1) for self-shrinkers.

Remark 2. Combining Theorem 2 with the upper bound estimate of weighted area for closed embedded $f$-minimal surfaces of fixed genus in a complete 3-manifold with $\overline{\operatorname{Ric}}_{f} \geq k>0$, we may obtain the smooth compactness theorem for the space of closed embedded $f$-minimal surfaces of fixed topological type and with diameter bound. We discuss it in [2].

\section{APPENDIX}

In this appendix, we discuss the $L_{f}$-stability properties of $f$-submanifolds. With the same notation as in Section 2, let $\left(M^{m}, \bar{g}\right)$ be an $m$-dimensional Riemannian manifold and $i: \Sigma^{n} \rightarrow M^{m}, n<m$, be an immersion. Let $\tilde{g}=e^{-\frac{2}{n} f} \bar{g}$ denote the new conformal metric on $M$. Therefore $i$ may induce two isometric immersions of $\Sigma:(\Sigma, \bar{g}) \rightarrow(M, \bar{g})$ and $(\Sigma, \tilde{g}) \rightarrow(M, \tilde{g})$ respectively.

When $(\Sigma, \tilde{g})$ is minimal, it is well known that the second variation of the volume of $(\Sigma, \tilde{g})$ is given by

Proposition 12 (cf. [6]). Let $(\Sigma, \tilde{g})$ be a minimal submanifold in $(M, \tilde{g})$. If $T$ is a normal compactly supported variational vector field on $\Sigma$ (that is, $T=T^{\perp}$ ), then the second variational formula of the volume $\tilde{V}$ of $(\Sigma, \tilde{g})$ is given by

$$
\left.\frac{d^{2}}{d t^{2}} \tilde{V}\left(\Sigma_{t}\right)\right|_{t=0}=-\int_{\Sigma}\langle T, J T\rangle_{\tilde{g}} d \tilde{\sigma},
$$

where the stability operator (or Jacobi operator) $J$ is defined on a normal vector field $T$ to $\Sigma$ by

$$
J T=\Delta_{(\Sigma, \tilde{g})}^{\perp} T+\operatorname{tr}_{(\Sigma, \tilde{g})}[\widetilde{R m}(\cdot, T) \cdot]^{\perp}+\tilde{B}(T)
$$


Here $\Delta_{(\Sigma, \tilde{g})}^{\perp} T=\sum_{i=1}^{n}\left(\nabla_{\tilde{e}_{i}}^{\perp} \nabla_{\tilde{e}_{i}}^{\perp} T-\nabla_{\nabla_{\tilde{e}_{i}} \tilde{e}_{i}}^{\perp} T\right)$ is the Laplacian determined by the normal connection $\nabla^{\perp}$ of $(\Sigma, \tilde{g}), \widetilde{R m}$ is the curvature tensor on $(M, \tilde{g}), \operatorname{tr}_{(\Sigma, \tilde{g})}[\widetilde{R m}(\cdot, T) \cdot]^{\perp}=$ $\sum_{i=1}^{n}\left[\widetilde{R m}\left(\tilde{e}_{i}, T\right) \tilde{e}_{i}\right]^{\perp}, \tilde{A}$ denotes the second fundamental form of $(\Sigma, \tilde{g}), \tilde{B}(T)=$ $\sum_{i, j=1}^{n}\left\langle\tilde{A}\left(\tilde{e}_{i}, \tilde{e}_{j}\right), T\right\rangle \tilde{A}\left(\tilde{e}_{i}, \tilde{e}_{j}\right)$, and $\left\{\tilde{e}_{i}\right\}, i=1, \cdots, n$, is a local orthonormal base of $(\Sigma, \tilde{g})$.

Recall that the weighted volume of $(\Sigma, \bar{g})$ is defined by

$$
V_{f}(\Sigma)=\int_{\Sigma} e^{-f} d \sigma
$$

By a direct computation similar to that of (20), we may prove the second variation formula of the weighted volume of $f$-minimal submanifold $(\Sigma, \bar{g})$.

Definition 5. For any normal vector field $T$ on $(\Sigma, \bar{g})$, the second order operator $\Delta_{f}^{\perp}$ is defined by

$$
\begin{aligned}
\Delta_{f}^{\perp} T & :=\Delta^{\perp} T-\operatorname{tr}\left[\nabla f \otimes \nabla^{\perp} T(\cdot, \cdot)\right] \\
& =\sum_{i=1}^{n}\left(\nabla_{e_{i}}^{\perp} \nabla_{e_{i}}^{\perp} T-\nabla_{\nabla_{e_{i}} e_{i}}^{\perp} T\right)-\sum_{i=1}^{n}\left(e_{i} f\right)\left(\nabla_{e_{i}}^{\perp} T\right) .
\end{aligned}
$$

The operator $L_{f}$ on $(\Sigma, \bar{g})$ is defined by

$$
L_{f} T=\Delta_{f}^{\perp} T+R(T)+B(T)+F(T) .
$$

In the above, $\nabla^{\perp}$ denotes the normal connection of $(\Sigma, \bar{g}) ;\left\{e_{i}\right\}, i=1, \ldots, n$, is a local orthonormal base of $(\Sigma, \bar{g}) ; B(T)=\sum_{i, j=1}^{n}\left\langle A\left(e_{i}, e_{j}\right), T\right\rangle A\left(e_{i}, e_{j}\right)$, where $A$ denotes the second fundamental form of $(\Sigma, \bar{g}) ; R(T)=\operatorname{tr}_{(\Sigma, \bar{g})}[\overline{R m}(\cdot, T) \cdot]^{\perp}=$ $\sum_{i=1}^{n}\left[\overline{R m}\left(e_{i}, T\right) e_{i}\right]^{\perp}$, where $\overline{R m}$ denotes the Riemannian curvature tensor of $(M, \bar{g})$; and $F(T)=\left[\bar{\nabla}^{2} f(T)\right]^{\perp}=\sum_{\alpha=n+1}^{m} \bar{\nabla}^{2} f\left(T, e_{\alpha}\right) e_{\alpha}$, where $\left\{e_{\alpha}\right\}, \alpha=n+1, \cdots, m$, is a local orthonormal normal vector field on $(\Sigma, \bar{g})$.

Proposition 13. Let $(\Sigma, \bar{g})$ be an f-minimal submanifold in $(M, \bar{g})$. If $T$ is a normal compactly supported variational vector field on $\Sigma$ (that is, $T=T^{\perp}$ ), then the second variation of the weighted volume of $(\Sigma, \bar{g})$ is given by

$$
\left.\frac{d^{2}}{d t^{2}} V_{f}\left(\Sigma_{t}\right)\right|_{t=0}=-\int_{\Sigma}\left\langle T, L_{f} T\right\rangle_{\bar{g}} e^{-f} d \sigma
$$

Proof. Let $\psi(\cdot, t), t \in(-\varepsilon, \varepsilon)$ be a compactly supported variation of $\Sigma$ so that $T=d \psi\left(\frac{\partial}{\partial t}\right)$ is the variational vector field, $\Sigma_{t}=\psi(\Sigma, t), \Sigma_{0}=\Sigma$. Choose a normal coordinate system $\left\{x_{1}, \ldots, x_{n}\right\}$ at a point $p \in \Sigma$. We can consider $\left\{x_{1}, \ldots, x_{n}, t\right\}$ to be a coordinate system of $\Sigma \times(-\varepsilon, \varepsilon)$ near the point $(p, 0)$. Denote $e_{i}=d \psi\left(\frac{\partial}{\partial x_{i}}\right)$ for $i=1, \ldots, n$. The induced metric on $\Sigma_{t}$ from $(M, \bar{g})$ is given for $g_{i j}=\left\langle e_{i}, e_{j}\right\rangle$. 
Hence $g_{i j}(p, 0)=\delta_{i j}$ and $\nabla_{e_{i}} e_{j}(p, 0)=0$. Denote by $d \sigma_{t}$ the volume element of $\Sigma_{t}$. Then $d \sigma_{t}=J(x, t) d \sigma_{0}$, where $d \sigma_{0}=d \sigma$ and the function $J(x, t)$ is given by

$$
J(x, t)=\frac{\sqrt{G(x, t)}}{\sqrt{G(x, 0)}},
$$

with $G(x, t)=\operatorname{det}\left(g_{i j}(x, t)\right)$. Denote by $d\left(\sigma_{f}\right)_{t}$ the weighted volume element of $\Sigma_{t}$. Then $d\left(\sigma_{f}\right)_{t}=J_{f}(x, t) d \sigma_{0}$, where $J_{f}(x, t)=J(x, t) e^{-f(x, t)}, f(x, t)=f(\psi(x, t))$.

Since $\frac{\partial J}{\partial t}=\sum_{i, j=1}^{n} g^{i j}\left\langle\bar{\nabla}_{e_{i}} T, e_{j}\right\rangle J, \frac{\partial J_{f}}{\partial t}=\left(\sum_{i, j=1}^{n} g^{i j}\left\langle\bar{\nabla}_{e_{i}} T, e_{j}\right\rangle-\langle\bar{\nabla} f, T\rangle\right) J_{f}$.

Note that $T$ is a normal vector field. A direct computation gives, at $(p, 0)$,

$$
\begin{aligned}
\left.\frac{\partial^{2} J_{f}}{\partial^{2} t}\right|_{t=0}= & {\left[-2 \sum_{i, j=1}^{n}\left\langle A_{i j}, T\right\rangle^{2}+\left\langle\bar{R}\left(e_{i}, T\right) T, e_{i}\right\rangle\right.} \\
& +\sum_{i=1}^{n}\left\langle\bar{\nabla}_{e_{i}} \bar{\nabla}_{T} T, e_{i}\right\rangle+\sum_{i=1}^{n}\left\langle\bar{\nabla}_{e_{i}} T, \bar{\nabla}_{e_{i}} T\right\rangle \\
& -\bar{\nabla}^{2} f(T, T)-\left\langle\bar{\nabla} f, \bar{\nabla}_{T} T\right\rangle \\
& \left.+\left(\sum_{i=1}^{n}\left\langle\bar{\nabla}_{e_{i}} T, e_{i}\right\rangle-\langle\bar{\nabla} f, T\rangle\right)\left(\sum_{j=1}^{n}\left\langle\bar{\nabla}_{e_{j}} T, e_{j}\right\rangle-\langle\bar{\nabla} f, T\rangle\right)\right] J_{f} .
\end{aligned}
$$

By

$$
\begin{aligned}
\sum_{i=1}^{n}\left\langle\bar{\nabla}_{e_{i}} T, \bar{\nabla}_{e_{i}} T\right\rangle & =\sum_{i, j=1}^{n}\left\langle\bar{\nabla}_{e_{i}} T, e_{j}\right\rangle^{2}+\sum_{i=1}^{n} \sum_{\alpha=n+1}^{m}\left\langle\bar{\nabla}_{e_{i}} T, e_{\alpha}\right\rangle^{2} \\
& =\sum_{i, j=1}^{n}\left\langle A_{i j}, T\right\rangle^{2}+\sum_{i=1}^{n}\left\langle\nabla_{e_{i}}^{\perp} T, \nabla_{e_{i}}^{\perp} T\right\rangle \\
& =|\langle A(\cdot, \cdot), T\rangle|^{2}+\left|\nabla^{\perp} T\right|^{2}
\end{aligned}
$$

and $\sum_{i=1}^{n}\left\langle\bar{\nabla}_{e_{i}} \bar{\nabla}_{T} T, e_{i}\right\rangle=\operatorname{div}\left(\bar{\nabla}_{T} T\right)^{\top}-\left\langle\left(\bar{\nabla}_{T} T\right)^{\perp}, \mathbf{H}\right\rangle$ we have that, at $p$,

$$
\begin{aligned}
\left.\frac{\partial^{2} J_{f}}{\partial t^{2}}\right|_{t=0}= & {\left[-|\langle A(\cdot, \cdot), T\rangle|^{2}-\sum_{i=1}^{n}\left\langle\bar{R}\left(e_{i}, T\right) e_{i}, T\right\rangle+\left|\nabla^{\perp} T\right|^{2}+\operatorname{div}\left(\bar{\nabla}_{T} T\right)^{\top}\right.} \\
& \left.-\left\langle\left(\bar{\nabla}_{T} T\right)^{\perp}, \vec{H}\right\rangle-\bar{\nabla}^{2} f(T, T)-\left\langle\bar{\nabla} f, \bar{\nabla}_{T} T\right\rangle+\left\langle T, \mathbf{H}_{f}\right\rangle^{2}\right] e^{-f}
\end{aligned}
$$

Using $\operatorname{div}\left(e^{-f}\left(\bar{\nabla}_{T} T\right)^{\top}\right)=e^{-f} \operatorname{div}\left(\bar{\nabla}_{T} T\right)^{\top}-e^{-f}\left\langle\left(\bar{\nabla}_{T} T\right)^{\top}, \nabla f\right\rangle$, we have at $p$ :

$$
\begin{aligned}
\left.\frac{\partial^{2} J_{f}}{\partial t^{2}}\right|_{t=0}= & {\left[\left|\nabla^{\perp} T\right|^{2}-|\langle A(\cdot, \cdot), T\rangle|^{2}-\sum_{i=1}^{n}\left\langle\bar{R}\left(e_{i}, T\right) e_{i}, T\right\rangle-\bar{\nabla}^{2} f(T, T)\right.} \\
& \left.-\left\langle\left(\bar{\nabla}_{T} T\right)^{\perp}, \mathbf{H}_{f}\right\rangle+\left\langle T, \mathbf{H}_{f}\right\rangle^{2}\right] e^{-f}+\operatorname{div}\left(e^{-f}\left(\bar{\nabla}_{T} T\right)^{\top}\right)
\end{aligned}
$$


Observe that the right-hand side of (25) is independent of the choice of coordinates. Hence (25) holds on $\Sigma$. By integrating (25) and using the fact that $\Sigma$ is $f$-minimal (i.e. $\mathbf{H}_{f}=0$ ), we obtain

$$
\begin{aligned}
\left.\frac{d^{2}}{d t^{2}} V_{f}\left(\Sigma_{t}\right)\right|_{t=0} & =\int_{\Sigma}\left(\left|\nabla^{\perp} T\right|^{2}-|\langle A(\cdot, \cdot), T\rangle|^{2}-\langle R(T), T\rangle-\bar{\nabla}^{2} f(T, T)\right) e^{-f} d \sigma \\
& =-\int_{\Sigma}\left\langle T, \Delta_{f}^{\perp} T+A(T)+R(T)+F(T)\right\rangle e^{-f} d \sigma \\
& =-\int_{\Sigma}\left\langle T, L_{f} T\right\rangle e^{-f} d \sigma .
\end{aligned}
$$

Substituting $e^{-f} T$ for $T$ in the identity $\int_{\Sigma}\left|\nabla^{\perp} T\right|^{2} d \sigma=-\int_{\Sigma}\left\langle T, \Delta^{\perp} T\right\rangle d \sigma$, we have

$$
\int_{\Sigma}\left|\nabla^{\perp} T\right|^{2} e^{-f} d \sigma=-\int_{\Sigma}\left\langle T, \Delta_{f}^{\perp} T\right\rangle e^{-f} d \sigma
$$

Thus we have the second variation formula of the weighted volume of $\Sigma$ :

$$
\begin{aligned}
\left.\frac{d^{2}}{d t^{2}} V_{f}\left(\Sigma_{t}\right)\right|_{t=0} & =-\int_{\Sigma}\left\langle T, \Delta_{f}^{\perp} T+A(T)+R(T)+F(T)\right\rangle e^{-f} d \sigma \\
& =-\int_{\Sigma}\left\langle T, L_{f} T\right\rangle e^{-f} d \sigma .
\end{aligned}
$$

Definition 6. An $f$-minimal submanifold $(\Sigma, \bar{g})$ is called $L_{f}$-stable if the second variation of the weighted volume of $\Sigma$ given by (24) is nonnegative for any normal compactly supported variational vector field $T$ on $\Sigma$.

Observe that for an $f$-minimal submanifold $\Sigma$ and its normal compactly supported variation, it holds that $V_{f}\left(\Sigma_{t}\right)=\tilde{V}\left(\Sigma_{t}\right)$. Then

$$
\left.\frac{d^{2}}{d t^{2}} \tilde{V}\left(\Sigma_{t}\right)\right|_{t=0}=\left.\frac{d^{2}}{d t^{2}} V_{f}\left(\Sigma_{t}\right)\right|_{t=0}
$$

By (20), (24), and (26), we have

$$
\int_{\Sigma}\langle T, J T\rangle_{\tilde{g}} d \tilde{\sigma}=\int_{\Sigma}\left\langle T, L_{f} T\right\rangle_{\bar{g}} e^{-f} d \sigma
$$

This implies that

$$
\int_{\Sigma} e^{-\frac{2 f}{n}}\langle T, J T\rangle_{\bar{g}} e^{-f} d \sigma=\int_{\Sigma}\left\langle T, L_{f} T\right\rangle_{\bar{g}} e^{-f} d \sigma
$$

By (28), the following equality holds.

Corollary 3. For any normal vector field $T$ on $\Sigma$,

$$
J T=e^{\frac{2 f}{n}} L_{f} T \text {. }
$$

The operator $L_{f}$ corresponds to a symmetric bilinear form $B_{f}(T, T)$ for the space of normal compactly supported vector fields on $\Sigma$ :

$$
B_{f}(T, T):=-\int_{\Sigma}\left\langle T, L_{f} T\right\rangle_{\bar{g}} e^{-f} d \sigma .
$$


We define the $L_{f}$-index, denoted by $L_{f}$-ind, of $(\Sigma, \bar{g})$ by the maximum of the dimensions of negative definite subspaces of $B_{f}$. Hence $(\Sigma, \bar{g})$ is $L_{f}$-stable if and only if its $L_{f}$-ind $=0$.

On the other hand, for minimal $(\Sigma, \tilde{g})$, it is well known that the stability operator $J$ also defines a symmetric bilinear form $\tilde{B}(T, T)$,

$$
\tilde{B}(T, T):=-\int_{\Sigma}\langle T, J T\rangle_{\tilde{g}} d \tilde{\sigma}
$$

There are also the concepts of index and stability of $(\Sigma, \tilde{g})$. In particular, $(\Sigma, \tilde{g})$ is stable if and only if the index $\operatorname{ind}(\Sigma, \tilde{g})=0$. Since $B_{f}(T, T)=\tilde{B}(T, T)$, it holds that

Proposition 14. $L_{f}$-ind of $(\Sigma, \bar{g})$ is equal to the index of $(\Sigma, \tilde{g})$. In particular, $(\Sigma, \bar{g})$ is $L_{f}$-stable if and only if $(\Sigma, \tilde{g})$ is stable in $(M, \tilde{g})$.

Now if $\Sigma$ is a two-sided hypersurface, that is, if there is a globally-defined unit normal $\nu$ on $(\Sigma, \bar{g})$, take $T=\varphi \nu$. Then the second variation (24) implies that

Proposition 15. Let $\Sigma$ be a two-sided $f$-minimal hypersurface in $\left(M^{n+1}, \bar{g}\right)$. If $\varphi$ is a compactly supported smooth function on $\Sigma$, then the second variation of the weighted volume of $(\Sigma, \bar{g})$ is given by

$$
\left.\frac{d^{2}}{d t^{2}} V_{f}\left(\Sigma_{t}\right)\right|_{t=0}=-\int_{\Sigma} \varphi L_{f}(\varphi) e^{-f} d \sigma
$$

where $\nu$ denotes the unit normal of $(\Sigma, \bar{g})$ and the operator $L_{f}$ is defined by $L_{f}=$ $\Delta_{f}+|A| \frac{2}{g}+\overline{\operatorname{Ric}}_{f}(\nu, \nu)$.

Definition 7. The operator $L_{f}=\Delta_{f}+|A| \frac{2}{g}+\overline{\operatorname{Ric}}_{f}(\nu, \nu)$ is called the $L_{f}$-stability operator of hypersurface $(\Sigma, \bar{g})$.

A bilinear form on space $C_{o}^{\infty}(\Sigma)$ of compactly supported smooth functions on $\Sigma$ is defined by

$$
\begin{aligned}
B_{f}(\varphi, \varphi): & =-\int_{\Sigma} \varphi L_{f} \varphi e^{-f} d \sigma \\
& =\int_{\Sigma}\left[|\nabla \varphi|^{2}-\left(|A|_{g}^{2}+\overline{\operatorname{Ric}}_{f}(\nu, \nu)\right) \varphi^{2}\right] e^{-f} d \sigma .
\end{aligned}
$$

The $L_{f}$-index, denoted by $L_{f}$-ind, of $(\Sigma, \bar{g})$ is defined to be the maximum of the dimensions of negative definite subspaces of $B_{f}$. Hence $(\Sigma, \bar{g})$ is $L_{f}$-stable if and only if $L_{f}$-ind $=0$. Clearly the definition of $L_{f}$-index is equivalent to the corresponding definition using the variational vector field $T$ as before.

Also, for minimal hypersurface $i:(\Sigma, \tilde{g}) \rightarrow\left(M^{n+1}, \tilde{g}\right)$, it is well known that if $\psi$ is a compactly supported smooth function on $\Sigma$, then the second variation of the volume $\tilde{V}$ of $\left(\Sigma, i^{*} \tilde{g}\right)$ is given by

$$
\left.\frac{d^{2}}{d t^{2}} \tilde{V}\left(\Sigma_{t}\right)\right|_{t=0}=-\int_{\Sigma} \psi J(\psi) d \tilde{\sigma}
$$


where $\tilde{A}$ denotes the second fundamental form of $(\Sigma, \tilde{g}), \tilde{\nu}$ denotes the unit normal of $(\Sigma, \tilde{g})$, and $J=\triangle_{\tilde{g}}+|\tilde{A}|_{\tilde{g}}^{2}+\widetilde{\operatorname{Ric}}(\tilde{\nu}, \tilde{\nu})$ is the stability operator (or the Jacobi operator) of $(\Sigma, \tilde{g})$.

The following holds, from (28).

Proposition 16. Let $\left(\Sigma^{n}, g\right)$ be an $f$-minimal hypersurface immersed in $(M, \bar{g})$. Then for all $\varphi \in \mathcal{C}_{o}^{\infty}(\Sigma)$,

$$
\int_{\Sigma}\left(e^{-\frac{f}{n}} \varphi\right) J\left(e^{-\frac{f}{n}} \varphi\right) e^{-f} d \sigma=\int_{\Sigma} \varphi L_{f}(\varphi) e^{-f} d \sigma
$$

Corollary 4. For $\varphi \in C^{\infty}(\Sigma), J\left(e^{-\frac{f}{n}} \varphi\right)=e^{\frac{f}{n}} L_{f}(\varphi)$.

Corollary 5. $L_{f}$-ind of $(\Sigma, \bar{g})$ is equal to the index of $(\Sigma, \tilde{g})$. In particular, $(\Sigma, \bar{g})$ is $L_{f}$-stable if and only if $(\Sigma, \tilde{g})$ is stable in $(M, \tilde{g})$.

\section{REFERENCES}

[1] Huai-Dong Cao and Detang Zhou, On complete gradient shrinking Ricci solitons, J. Differential Geom. 85 (2010), no. 2, 175-185. MR2732975 (2011k:53040)

[2] Xu Cheng, Tito Mejia, and Detang Zhou, Eigenvalue estimate and compactness for closed f-minimal surfaces, Pacific J. Math. 271 (2014), no. 2, 347-367, DOI 10.2140/pjm.2014.271.347. MR 3267533

[3] Xu Cheng and Detang Zhou, Volume estimate about shrinkers, Proc. Amer. Math. Soc. 141 (2013), no. 2, 687-696, DOI 10.1090/S0002-9939-2012-11922-7. MR 2996973

[4] Tobias H. Colding and William P. Minicozzi II, Smooth compactness of self-shrinkers, Comment. Math. Helv. 87 (2012), no. 2, 463-475, DOI 10.4171/CMH/260. MR2914856

[5] Tobias H. Colding and William P. Minicozzi II, Generic mean curvature flow I: generic singularities, Ann. of Math. (2) 175 (2012), no. 2, 755-833, DOI 10.4007/annals.2012.175.2.7. MR2993752

[6] Tobias Holck Colding and William P. Minicozzi II, A course in minimal surfaces, Graduate Studies in Mathematics, vol. 121, American Mathematical Society, Providence, RI, 2011. MR2780140

[7] Tobias H. Colding and William P. Minicozzi II, Estimates for parametric elliptic integrands, Int. Math. Res. Not. 6 (2002), 291-297, DOI 10.1155/S1073792802106106. MR1877004 (2002k:53060)

[8] Tobias H. Colding and William P. Minicozzi II, Embedded minimal surfaces without area bounds in 3-manifolds, Geometry and topology: Aarhus (1998), Contemp. Math., vol. 258, Amer. Math. Soc., Providence, RI, 2000, pp. 107-120, DOI 10.1090/conm/258/04058. MR.1778099 (2001i:53012)

[9] Qi Ding and Y. L. Xin, Volume growth, eigenvalue and compactness for self-shrinkers, Asian J. Math. 17 (2013), no. 3, 443-456, DOI 10.4310/AJM.2013.v17.n3.a3. MR.3119795

[10] Frank Morgan, Manifolds with density, Notices Amer. Math. Soc. 52 (2005), no. 8, 853-858. MR2161354(2006g:53044)

[11] Ovidiu Munteanu and Jiaping Wang, Analysis of weighted Laplacian and applications to Ricci solitons, Comm. Anal. Geom. 20 (2012), no. 1, 55-94, DOI 10.4310/CAG.2012.v20.n1.a3. MR2903101

[12] Ovidiu Munteanu and Jiaping Wang, Geometry of manifolds with densities, Adv. Math. 259 (2014), 269-305, DOI 10.1016/j.aim.2014.03.023. MR3197658

[13] Guofang Wei and Will Wylie, Comparison geometry for the Bakry-Emery Ricci tensor, J. Differential Geom. 83 (2009), no. 2, 377-405. MR2577473(2011a:53064) 
[14] B. White, Curvature estimates and compactness theorems in 3-manifolds for surfaces that are stationary for parametric elliptic functionals, Invent. Math. 88 (1987), no. 2, 243-256, DOI 10.1007/BF01388908. MR880951 (88g:58037)

Instituto de Matematica e Estatística, Universidade Federal Fluminense, Niterói, RJ 24020, BRAZIL

E-mail address: xcheng@impa.br

Instituto de Matematica e Estatística, Universidade Federal Fluminense, Niterói, RJ 24020, BRAZIL

E-mail address: tmejia.uff@gmail.com

Instituto de Matematica e Estatística, Universidade Federal Fluminense, Niterói, RJ 24020, BRAZIL

E-mail address: zhou@impa.br 